Research Paper

\title{
Temporomandibular Disorders in Burning Mouth Syn- drome Patients: An Observational Study
}

\author{
Massimo Corsalini ${ }^{1 凶}$, Daniela Di Venere ${ }^{2}$, Francesco Pettini ${ }^{3}$, Dorina Lauritano ${ }^{4}$, Massimo Petruzzi $^{5}$ \\ 1. MD, DMD Assistant professor, Dental School University of Bari, Section of TMD Diagnosis and Treatment \\ 2. DDS, PhD, PG Orth Assistant professor, Dental School University of Bari, Section of TMD Diagnosis and treatment \\ 3. DDS, Assistant professor, Dental School University of Bari, Section of Restorative Dentistry \\ 4. DDS, Assistant professor, Dental School University of Milan Bicocca, Section of Oral Pathology and Medicine \\ 5. DDS, PhD, PG Oral.Surg. Assistant professor, Dental School University of Bari, Section of Oral Pathology and Medicine
}

$\square$ Corresponding author: Prof. Massimo Corsalini. Clinica odontoiatrica. Università degli Studi di Bari, Piazza Giulio Cesare 11, 70124 Bari. Tel. +39 0805478729. Fax. +39080 5478743. massimo.corsalini@uniba.it

(c) Ivyspring International Publisher. This is an open-access article distributed under the terms of the Creative Commons License (http://creativecommons.org/ licenses/by-nc-nd/3.0/). Reproduction is permitted for personal, noncommercial use, provided that the article is in whole, unmodified, and properly cited.

Received: 2013.03.25; Accepted: 2013.10.07; Published: 2013.10.29

\begin{abstract}
BACKGROUND: Burning Mouth Syndrome (BMS) is a chronic disease characterized by absence of any lesions and burning of the oral mucosa associated to a sensation of dry mouth and/or taste alterations. The purpose of our study is to estimate signs and symptoms of Temporomandibular Disorders (TMD) in patients with BMS and to investigate for the existence of an association between BMS and TMD.

MATERIALS AND METHODS: Forty-four BMS patients were enrolled; BMS subtype was established according to the classification of Lamey. After a gnathological evaluation, according to the protocol of the European Academy of Craniomandibular Disorders, patients were classified by RDC/TMD criteria. The data were compared and analyzed using a chi-square test to describe the existence of an association between BMS and TMD.

RESULTS: $65.9 \%$ the BMS patients showed disorders classified as primary signs and symptoms of TMD according to RDC/TMD criteria, and $72.7 \%$ showed parafunctional habits. The chi-square test revealed a statistically significant association $(p=0.035)$ between BMS and TMD.

CONCLUSION: The data suggest that there is a possible relationship not yet well understood between BMS and TMD, may be for neurophatic alterations assumed for BMS that could be also engaged in TMD pathogenesis.
\end{abstract}

Key words: TMD, BMS, parafunctional habits, oral pain, RDC/TMD criteria

\section{Introduction}

Burning mouth syndrome has been defined as burning pain in the tongue or oral mucous membranes without accompanying clinical and laboratory findings (1).

The prevalence of BMS has been reported to be between $0.7 \%$ and $5.0 \%$ of the general population (2). A female predominance (female to male ratio of 3:1 to $7: 1)$ is seen in a pair of studies, with lower ratios found in population-based studies $(3,4)$.

The etiopathogenesis of BMS is still unclear, and the issue has generated considerable controversy in the literature (5).

Scala et al. distinguished two different types of BMS: the primary and the secondary one (6). The primary BMS is the "true" idiopathic BMS while in the secondary BMS it is possible to relate the burning sensation to local conditions (infections, allergic reactions, galvanism, geographic tongue, dental treatment) or systemic diseases (menopausal disorders, diabetes mellitus thyroid dysfunctions, nutritional 
deficiencies). Jääskeläinen proposed to consider three different subgroups of primary BMS patients: Each subgroup shows different pathogenetic mechanism responsible of the BMS. The first BMS patient subgroup $(50 \%-65 \%)$ is characterized by peripheral small diameter fiber neuropathy of intraoral mucosa; the second subgroup ( $20 \%-25 \%$ of BMS patients) consists of patients with subclinical lingual, mandibular or trigeminal system pathology that can be dissected with careful neurophysiologic examination, but is clinically indistinguishable from the other 2 subgroups. The third subgroup $(20 \%-40 \%)$ may suffer of a hypofunction of dopaminergic neurons in the basal ganglia (7).

About clinical features, the intensity of burning in BMS ranges from moderate to severe and it is usually less severe in the morning and during the mastication (2).

Lamey and Lewis (8) classified BMS into three different types: type I, with symptoms not present upon awakening in the morning but starting during the day; type II, with burning present upon awakening in the morning and persisting throughout the day; and type III, with intermittent symptoms in the whole day.

The burning is bilateral in more cases and does not respect the anatomic distribution of the peripheral nerves (it is typically reported to occur at more than one site) (9). The tongue is the most affected site, followed by the lips, palate, and cheeks.

Symptom onset is usually spontaneous, with no identifiable precipitating factors and once it starts, the burning pain may last for several years (2). The rate of spontaneous remission is only about $3 \%$ over a 5 -year period $(2,5,8,10,11)$.

A definitive treatment for BMS is not yet available: BMS patients have shown a good response to a long-term therapy with antidepressants (12) and anxiolytics (13).

Some patients undergoing topical or systemic capsaicin administration have experienced a partial or even complete remission of their pain $(14,15)$.

Psychological disorders are detected in many patients, so a psychodynamic therapy has been proposed (16-18). There are studies about alpha lipoic acid as neuroprotective factor $(19,20)$ but some Authors do not agree to its use in BMS treatment (21). More recent studies have demonstrated the efficacy of clonazepam (topical or systemic) alone or associated to psychotherapy and the effectiveness of duloxetine in the treatment of atypical facial pain (22-24).

Temporomandibular Disorders (TMD) are a group of clinical diseases that involve the masticatory muscles, the temporomandibular joint (TMJ) or both. The most common signs and symptoms of TMD are orofacial pain, several types of TMJ sounds elicited by mandibular excursions, tenderness of the muscles of mastication and the TMJ, restricted range of mandibular motion. The Research Diagnostic Criteria in Axis I divided the clinical TMD conditions into three groups: Muscle diagnoses (myofascial pain with or without limited opening), disc displacements (with reduction, without reductions with limited openings, without reduction without limited opening) and arthralgia, arthritis and arthrosis (25).

Similarly to BMS, TMD are quite common in the general population in fact population studies have reported the prevalence of TMD to be from $8 \%$ to $15 \%$ for women and from $3 \%$ to $10 \%$ for men, suggesting that TMD are significant causes of pain in the head and face region (26). While the etiology of TMD is still not well described, in the literature, it is generally thought that they are conditions comprising both psychosocial and neurophysiologic entities (27).

Temporomandibular pain may also be associated with sensorial disturbances when the pain is chronic and induces sensitization $(28,29)$. These alterations are not only somatosensory, but also gustative and olfactory (30).

To date, there is no definitive hypothesis that explains the coexistence of BMS and TMD. The aim of this observational study is to describe the characteristics of TMD (signs and symptoms) in patients with BMS and to evaluate the coexistence prevalence of the two diseases. We also aim to discriminate which of the wide clinical aspects of TMD (myofascial pain, internal derangements or degenerative joint diseases) are more frequently associated to the burning sensation.

\section{MATERIALS AND METHODS}

\section{Patient recruitment}

Since April 2008 to December 2008, consecutive patients with a confirmed diagnosis of BMS referred to Dental Clinic of University of Bari (Section of oral pathology and medicine) were included in the present observational study.

\section{Sample size calculation}

The appropriate sample size was determined evaluating three factors: (i) the estimated prevalence of BMS in the general population (p), (ii) a level of confidence of $95 \%(t)$ and (iii) a margin of error at $5 \%$ $(m)$. The sample size $(n)$ was calculated according to the following formula:

$$
n=t^{2 *} p(1-p) / m^{2}
$$

\section{BMS diagnosis}

Inclusion criterion was a persistent idiopathic 
mucosal oral burning sensation for at least 4- 6 months, in absence of any oral mucosal macroscopic lesions. Normal salivary flow rates and absence of bacterial or fungal infections were considered for BMS diagnosis. Laboratory analyses included hematological assessment of nutritional deficiencies, blood glucose levels, patch testing for specific allergies. None of enrolled patients were previously diagnosed nor treated for BMS and/or TMD.

\section{TMD evaluation}

A clinical and instrumental TMJ evaluation was performed, according to the protocol of the European Academy of Craniomandibular Disorders (E.A.C.D.). This protocol consists of an anamnestic questionnaire and of an accurate clinical examination. The anamnestic questionnaire concerns the patient's general health and the past and current presence of symptoms of TMD, as pain and/or fatigue in the muscles of mastication, TMJ pain or dysfunction, limitation of mandibular movements. Subjective perception of pain was recorded using a visual analogue scale (VAS). The site of pain was indicated by patient using the hand and was reported in the clinical folder. The presence of self-reported parafunctional habits (such as tongue indentations) and associated etiological factors were investigated.

Then patients were subjected to clinical trials and orthopedic tests to investigate dysfunctions during mandibular dynamics, pain during jaw movements or post palpation on masticatory muscles and/or TMJ, wear facets, TMJ noises. Lastly patients have been classified by Dworkin and LeResche RDC / TMD criteria (25).

\section{Statistical analysis}

Statistical methods included descriptive statistics of the cohort and the chi-square test to evaluate the correlation between BMS and TMD. The data obtained were analyzed using the SPSS program (Version 12.0 for Windows, SPSS Inc., Chicago, Ill).

\section{Ethical Approval}

The study was conducted according to the Declaration of Helsinki and was approved by ethical committee of Dental Sciences Department of Bari University. Every patient gave written informed consent to the study.

\section{RESULTS}

Forty-four consecutive patients affected by BMS, six male $(12.1 \%)$ and thirty-eight female $(87.9 \%)$ aged between 45 and 89 years (mean age $67 \pm 10.5$ ) were enrolled. Twenty-five patients $(56.8 \%)$ were affected by BMS type I, fifteen patients (34.1\%) by BMS type II and the remaining four $(9.1 \%)$ by BMS type III according to Lamey's classification (4). The mean intensity of burning evaluated by VAS was $5.5 \pm 1.8$ considering all the enrolled patients. Patients affected by type I BMS presented a mean VAS of $5.6 \pm 1.9$ patients affected by type II BMS presented a mean VAS of $5.7 \pm$ 2.1 and patients suffering from type III BMS showed a mean VAS of $4.5 \pm 0.5$.

Twenty patients $(45.5 \%)$ reported a significant facial pain different from burning. The most common site of pain was the masseter region (seven cases); then the head and neck area. Less involved areas were the medial pterygoids and the TMJ region. The mean VAS score for the facial pain was $2.4 \pm 1.6$.

Thirty-two patients $(72.7 \%)$ showed parafunctional habits. In particular night bruxism was observed in twenty-eight cases, clenching in ten cases, biting of lips and cheeks in six cases. In nine patients $(20.4 \%)$ were found limitation in mandibular movements associated to lateral movements (five cases), protraction and lateral movements (one case), opening and lateral movements (two cases) and protraction and lateral movements (one case). Only two patients showed a deviation to the right side of the mandible during opening and closing movements. Only in four patients the endfeel was inelastic.

In fifteen patients (34.1\%) was heard a clicking sound during both opening and closing mandibular movements (the so-called reciprocal click): in eight patients this sound was bilateral, in the remaining seven was unilateral. A crackling sound was heard only in one case.

The Joint play test was positive in seven cases.

On palpating the TMJ, three patients referred pain in the lateral side of the mandible condyle and other two patients in the lateral and back side.

The Joint muscle test was positive in seven cases. In particular only a patient had pain on static testing; the remaining six had noises on both static and dynamic testing. tient.

Compression test was positive only in one pa-

Twenty-two patients (50\%) were painful on palpation of the muscles of mastication.

The most affected muscles were medial pterygoids (twenty-one cases) and masseters (sixteen cases). Less involved were suprahyoids (six cases), neck (three cases), temporals (two cases), sternocleidomastoid muscle and shoulders (one case). $(77.2 \%)$.

Wear facets were observed in thirty-four patients

In twenty-nine patients (65.9\%) it was possible to notice disorders classified as primary signs and/or symptoms of TMD on the basis of RDC/TMD criteria.

In particular ten patients $(22.7 \%)$ were classified 
as " $1 \mathrm{a}$ " and one patient as " $1 \mathrm{~b}$ "; ten patients were classified as " $2 a$ ", three patients $(6.8 \%)$ as " $1 \mathrm{a}+2 \mathrm{a}$ " and one patient as " $1 \mathrm{a}+3 \mathrm{a}$ " One patient was classified as " $2 a+3 a$ ", and three patients as " $3 c$ ".

Then fifteen patients had myofascial pains, fourteen patients had internal derangements while five patients had degenerative joint diseases.

Fifteen patients $(34.1 \%)$ show no signs and / or symptoms of TMD so they have been classified like “ 0 ".

Figure 1 summarizes the distribution of the sample according to the type of dysfunction found for each patient.

Myofascial pains were frequently found in patients affected by BMS I while internal derangements in patients affected by BMS II.

Figure 2 summarizes the distribution of the sample according to the three typology of BMS and the type of dysfunction found for each patient.

Table 1 shows the results of chi-square test. The test revealed a statistically significant association $(p<.05)$ between BMS and TMD. A statistically significance was also found for the absence of degenerative joint diseases in patients affected by BMS while there was no statistically significance for the variables "myofascial pain" and "internal derangements".

Table I. Statistical analysis between BMS and dependent variables for TMD.

\begin{tabular}{ccc}
\hline & Dependent variables & p value \\
\hline & Total of TMD found & $\mathbf{p}=\mathbf{0 . 0 3 5}$ \\
29 & \\
BMS patients & Myofascial pain & $\mathrm{p}=0.853$ \\
$(44)$ & 15 & \\
& Internal derangements & $\mathrm{p}=0.853$ \\
14 & $\mathbf{p}=\mathbf{0 . 0 0 1}$ \\
& Degenerative joint diseases & \\
5
\end{tabular}

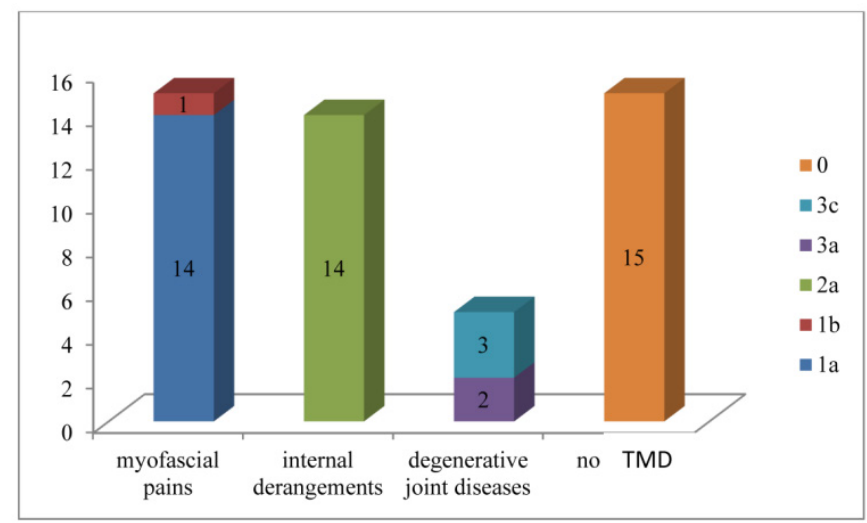

Figure I. Distribution of the sample according to the type of dysfunction found for each patient.

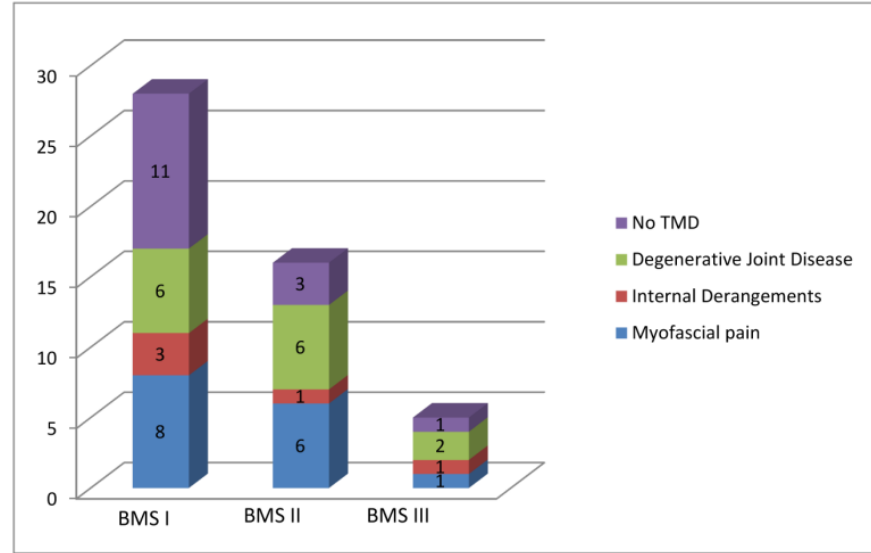

Figure 2. Distribution of the sample according to the three typology of BMS and the type of dysfunction founded for each patient.

\section{DISCUSSION}

The results of this observational study evidence that about two thirds of BMS patients showed primary signs and symptoms of TMD according to the protocol of the European Academy of Craniomandibular Disorders. Myofascial pain and internal derangements are the most common observed TMD and their prevalence is related to the BMS type. To the best of our knowledge, this is the first observational study that utilizes the protocol of the European Academy of Craniomandibular Disorders in patients suffering from BMS. In fact, different Authors have investigated the relationship between BMS and parafunctional habits or temporomandibular joint diseases. Lamey and Lamb (4) found the presence of parafunctional habits in $20.6 \%$ of the BMS patients examined; in the Paterson et al. study this percentage rising to $61 \%$ (10). On the contrary, López-Jornet et al., did not record significant differences in terms of parafunctional habits in a cohort of sixty BMS patients (31). According to the negative association between BMS and parafunctions are Mendak-Ziółko et al. In their multifactor analysis with logistic regression, they did not identify parafunctions as significant risk factor for BMS (32). Bergdahl et al., evaluated thirty patients resistant to BMS treatment: TMJ, masticatory muscle and tongue dysfunctions were frequently found (16). They concluded that "in some cases, resistant BMS probably is of psychological origin". Svensson and Kaaber (33) observed a higher frequency of pain/ weakness in masticatory, neck, shoulder, and suprahyoid muscles in patients with BMS when compared with healthy subjects. However, to date, no author has reported extensive data concerning the characteristics of the symptoms nor have qualitative/quantitative evaluations of mandibular dynamics movements been carried out.

Moreover no patients had previously undergone 
a gnathological treatment, nor felt it necessary because concern about the primary disease (BMS) probably made these patients miss or ignore signs and symptoms of TMD.

This also accords with the results of a Goulet's study, that reported only $2 \%$ of these patients require a gnathological approach (34).

The high percentage of TMD founded in BMS patients could be due to an overload of the masticatory system: anxiety and restlessness were frequently referred in the anamnestic interview and wear facets were often observed during the clinical evaluation of these patients as an effect of the stress associated to the primary disease.

As it known wear facet are often the initial indicators of parafunctional habits and in fact thirty-two patients $(72.7 \%$ of the sample) showed at least one parafunctional habit.

Probably, the stress condition associated to the primary disease could explain in these patients the onset of parafunctional habits, main causes of TMD.

However it is also possible that the same neuropathic alterations assumed for the BMS, could be engaged in parafunctional habits too. Lauria (35) showed a device trigeminal sensory neuropathy in patients affected by BMS.

This neuropathy results from a nigrostriatal dopaminergic system dysfunction that affects nociception regulation, causing a complete loss of inhibition of the trigeminal system. This would consist of a sensory and motor hyperfunction and then a masticatory muscles hyperactivity with onset of TMD.

Nerve fiber injury may escalate over time leading to a complete nerve degeneration. This could explain why myofascial pains and internal derangements were frequently found than degenerative joint diseases: degenerative joint diseases require a longer-term onset then myofascial pains and internal derangements and the complete nerve degeneration and then sensory and motor hyperfunction ending could occur before they can arise (30).

Further randomized control trials and metanalytic studies are necessary to evaluate the relationship between BMS and TMD.

\section{Competing Interests}

The authors have declared that no competing interest exists.

\section{References}

1. Merskey H, Bogduk N. Classification of chronic pain: descriptions of chronic pain syndromes and definitions of pain terms/prepared by the Task Force on Taxonomy of the International Association for the Study of Pain. $2 \mathrm{~d}$ ed. Seattle: IASP, 1994:742.

2 Grushka M, Epstein J, Gorsky M. Burning mouth syndrome. Am Fam Physician. 2002;65:615-620
3. Lipton JA, Ship JA, Larach-Robinson D. Estimated prevalence and distribution of reported orofacial pain in the United States. J Am Dent Assoc. 1993; 124: 115-121.

4. Lamey PJ, Lamb AB. Prospective study of aetiological factors in burning mouth syndrome. Br Med J (Clin Res Ed). 1988; 296: 1243-1246.

5. Danhauer SC, Miller CS, Rhodus NL, et al. Impact of criteria-based diagnosis of burning mouth syndrome on treatment outcome. J Orofac Pain. 2002; 16:305-311.

6. Scala A, Checchi L, Montevecchi M, et al. Update On Burning Mouth Syndrome: Overview and patient management. Crit Rev Oral Biol Med. 2003; 14: 275-291.

7. Jääskeläinen SK. Pathophysiology of primary burning mouth syndrome. Clin Neurophysiol. 2012; 123: 71-7.

8. Lamey PJ, Lewis MAO. Oral medicine in practice: burning mouth syndrome. Br Dent J. 1989; 167: 197- 200

9. Jääskeläinen SK. Clinical neurophysiologic and quantitative sensory testing in the investigation of orofacial pain and sensory function. J Orofac Pain. 2004; 18. 85-107.

10. Paterson AJ, Lamb AB, Clifford TJ, et al. Burning mouth syndrome: the relationship between the HAD scale and parafunctional habits. J Oral Pathol Med. 1995; 24: 289-292.

11. Sardella A, Lodi G, Demarosi F, Bez C, Cassano S, Carrassi A. Burning mouth syndrome: a retrospective study investigating spontaneous remission and response to treatments. Oral Dis. 2006; 12(2): 152-5

12. Maina G, Vitalucci A, Grandolfo S, et al. Comparative efficacy of SSRIs and amisulpride in burning mouth syndrome: a single-blind study. J Clin Psychiatry. 2002; 63:38-43.

13. Grushka M, Epstein J, Mott A. An open-label, dose escalation pilot study of the effect of clonazepam in burning mouth syndrome. Oral Surg Oral Med Oral Pathol Oral Radiol Endod. 1998; 86: 557-561.

14. Epstein J and Marcoe JH. Topical application of capsaicin for treatment of noral neuropathic pain and trigeminal neuralgia. Oral Surg Oral Med Oral Pathol. 1994; 77:135-40

15. Petruzzi M, Lauritano D, De Benedittis M, et al. Systemic capsaicin for burning mouth syndrome: short-term results of a pilot study. J Oral Pathol Med. 2004; 33:111-4.

16. Bergdahl J, Anneroth G, Perris H. Cognitive therapy in the treatment of patients with resistant burning mouth syndrome: a controlled study. J Oral Pathol Med. 1995; 24: 213-215.

17. Van Houdenhove B, Joostens P. Burning mouth syndrome. Successful treatment with combined psychotherapy and psychopharmacotherapy. Gen Hosp Psychiatry $1995 \cdot 17: 385-388$

18. Humphris GM, Longman LP, Field EA. Cognitive-behavioural therapy for idiopathic burning mouth syndrome: a report of two cases. Br Dent J. 1996; 181: 204-208.

19. Femiano F, Gombos F, Scully C, et al.. Burning mouth syndrome (BMS): controller open trial of the efficacy of alpha-lipoic acid (thioctic acid) on symptomatology. Oral Dis. 2000; 6: 274-7.

20. Femiano F, Scully C. Burning mouth syndrome (BMS): double blind controlled study of alpha-lipoic acid (thioctic acid) therapy. J Oral Pathol Med. 2002; 31: 267-269.

21. Cavalcanti DR, da Silveira FR. Alpha lipoic acid in burning mouth syndrome A randomized double-blind placebo-controlled trial. J Oral Pathol Med. 2009; 38: 254-261.

22. Rodríguez de Rivera Campillo E, López-López J, Chimenos-Küstner E. Response to topical clonazepam in patients with burning mouth syndrome: a clinical study. Bull Group Int Rech Sci Stomatol Odontol. 2010; 49(1): 19-29.

23. Rodríguez-de Rivera-Campillo E, López-López J. Evaluation of the response to treatment and clinical evolution in patients with burning mouth syndrome. Med Oral Patol Oral Cir Bucal. 2013; 18(3): 403-10.

24. Nagashima W, Kimura H, Ito M, Tokura T, Arao M, Aleksic B, Yoshida K, Kurita K, Ozaki N. Effectiveness of duloxetine for the treatment of chronic nonorganic orofacial pain. Clin Neuropharmacol. 2012; 35 :273-7.

25. Dworkin SF, LeResche L. Research diagnostic criteria for temporomandibular disorders: review, criteria, examinations and specifications, critique. J Craniomandib Disord. 1992: 6 301-355.

26. LeResche L. Epidemiology of temporomandibular disorders: Implications for the investigation of etiologic factors. Crit Rev Oral Biol Med. 1997; 8: 291-305.

27. Suvinen TI, Reade PC,Kemppainen P,Kononen M, Dworkin SF. Review of aetiological concepts of temporomandibular pain disorders: Towards a biopsychosocial model for integration of physical disorder factors with psychological and psychosocial illness impact factors. Eur I Pain. 2005; 9: 613-633.

28. Ayesh EE, Jensen TS, Svensson P. Hypersensitivity to mechanical and intra-articular electrical stimuli in persons with painful temporomandibular joints. J Dent Res. 2007; 86: 1187-1192.

29. Pfau DB, Rolke R, Nickel R, Treede RD, Daublaender M. Somatosensory profiles in subgroups of patients with myogenic temporomandibular disorders and fibromyalgia syndrome. Pain. 2009; 147: 72-83.

30. Grushka M, Epstein JB, Gorsky M. Burning mouth syndrome and other oral sensory disorders: a unifying hypothesis. Pain Res Manag. 2003; 8: 133-135.

31. López-Jornet P, Camacho-Alonso F, Leon-Espinosa S. Burning mouth syndrome, oral parafunctions, and psychological profile in a longitudinal case study. J Eur Acad Dermatol Venereol. 2009; 23(3): 363-5. 
32. Mendak-Ziółko M, Konopka T, Bogucki ZA. Evaluation of select neurophysiological, clinical and psychological tests for burning mouth syndrome. Oral Surg Oral Med Oral Pathol Oral Radiol. 2012; 114(3):325-32.

33. Svensson P, Kaaber S. General health factors and denture function in patients with burning mouth syndrome and matched control factors. J Oral Rehabil. 1995; 22: 887-895.

34. Goulet JP, Lund JP, Montplaisir J, Lavigne G. Daily clenching,nocturnal bruxism, and stress and their association with TMD symptoms. J Orofacial Pain. 1993; 7:120-127.

35. Lauria G, Majorana A, Borgna M, Lombardi R, Penza P, Padovani A, Sapelli P.

Trigeminal small-fiber sensory neuropathy causes burning mouth sindrome. Pain. 2005; 115: 332-337. 